\title{
Spinal Cord Injury after Blunt Cervical Spine Trauma: Correlation of Soft-Tissue Damage and Extension of Lesion
}

\author{
R. Martínez-Pérez, I. Paredes, S. Cepeda, A. Ramos, A.M. Castaño-León, C. García-Fuentes, R.D. Lobato, P.A. Gómez, and A. Lagares
}

\begin{abstract}
BACKGROUND AND PURPOSE: In patients with spinal cord injury after blunt trauma, several studies have observed a correlation between neurologic impairment and radiologic findings. Few studies have been performed to correlate spinal cord injury with ligamentous injury. The purpose of this study was to retrospectively evaluate whether ligamentous injury or disk disruption after spinal cord injury correlates with lesion length.
\end{abstract}

MATERIALS AND METHODS: We retrospectively reviewed 108 patients diagnosed with traumatic spinal cord injury after cervical trauma between 1990-2011. Plain films, CT, and MR imaging were performed on patients and then reviewed for this study. MR imaging was performed within 96 hours after cervical trauma for all patients. Data regarding ligamentous injury, disk injury, and the extent of the spinal cord injury were collected from an adequate number of MR images. We evaluated anterior longitudinal ligaments, posterior longitudinal ligaments, and the ligamentum flavum. Length of lesion, disk disruption, and ligamentous injury association, as well as the extent of the spinal cord injury were statistically assessed by means of univariate analysis, with the use of nonparametric tests and multivariate analysis along with linear regression.

RESULTS: There were significant differences in lesion length on T2-weighted images for anterior longitudinal ligaments, posterior longitudinal ligaments, and ligamentum flavum in the univariate analysis; however, when this was adjusted by age, level of injury, sex, and disruption of the soft tissue evaluated (disk, anterior longitudinal ligaments, posterior longitudinal ligaments, and ligamentum flavum) in a multivariable analysis, only ligamentum flavum showed a statistically significant association with lesion length. Furthermore, the number of ligaments affected had a positive correlation with the extension of the lesion.

CONCLUSIONS: In cervical spine trauma, a specific pattern of ligamentous injury correlates with the length of the spinal cord lesion in MR imaging studies. Ligamentous injury detected by MR imaging is not a dynamic finding; thus it proved to be useful in predicting neurologic outcome in patients for whom the MR imaging examination was delayed.

ABREVIATIONS: $\mathrm{ALL}=$ anterior longitudinal ligament; $\mathrm{ASIA}=$ American Spinal Injury Association Impairment Scale; $\mathrm{LF}=$ ligamentum flavum; $\mathrm{PLL}=$ posterior longitudinal ligament; $\mathrm{SCI}=$ spinal cord injury; SCIWORA = spinal cord injury without radiological abnormalities

A cute traumatic spinal cord injury (SCI) is located at the cervical level in $45-75 \%$ of cases. ${ }^{1,2}$ Thus, cervical trauma is potentially the source of long-term disability because of its associated risk of SCI. ${ }^{2}$ The internal architecture of the spinal cord, as well as soft tissue injury, is best visualized with MR imaging. However, MR imaging performed after the cervical trauma has prognostic implications for the therapeutic management of this disease; hence, it has become part of the standard imaging protocol

Received February 5, 2013; accepted after revision September 9.

From the Departments of Neurosurgery (R.M.-P., I.P., S.C., AM.C.-L., R.D.L., P.A.G., A.L.) and Radiology (A.R.), and Intensive Care Unit (C.G.-F.), Hospital 12 de Octubre, Universidad Complutense de Madrid, Madrid, Spain.

Please address correspondence to Rafael Martínez-Pérez, MD, Hospital 12 de Octubre. Andalucía s/n, 28041, Madrid, Spain; e-mail: rafallsafin@hotmail.com

http://dx.doi.org/10.3174/ajnr.A3812 for patients with acute cervical spine injury. ${ }^{3}$ Since the early 1990 s, several studies have investigated the role of several radiologic findings in SCI. ${ }^{3,4}$ Previous published reports have described the mechanism and biomechanics of spinal stability after blunt trauma. ${ }^{5-7}$ It is believed that the degree of soft-tissue damage is related to the severity of the SCI. However, very few studies have similarly shown this association.

Instead, the correlation between lesion length and neurologic outcome has been sufficiently demonstrated in the acute phase after cervical trauma. ${ }^{8,9}$ In fact, it has been recommended that the first MR imaging should be performed 24-72 hours after injury. ${ }^{10}$ The prognostic value of MR imaging in later stages is less clear because of the dynamic nature of edema and the dependence on the lesion length after trauma. ${ }^{11}$ Therefore, we designed this study to detect soft-tissue injuries that are related to more pronounced 

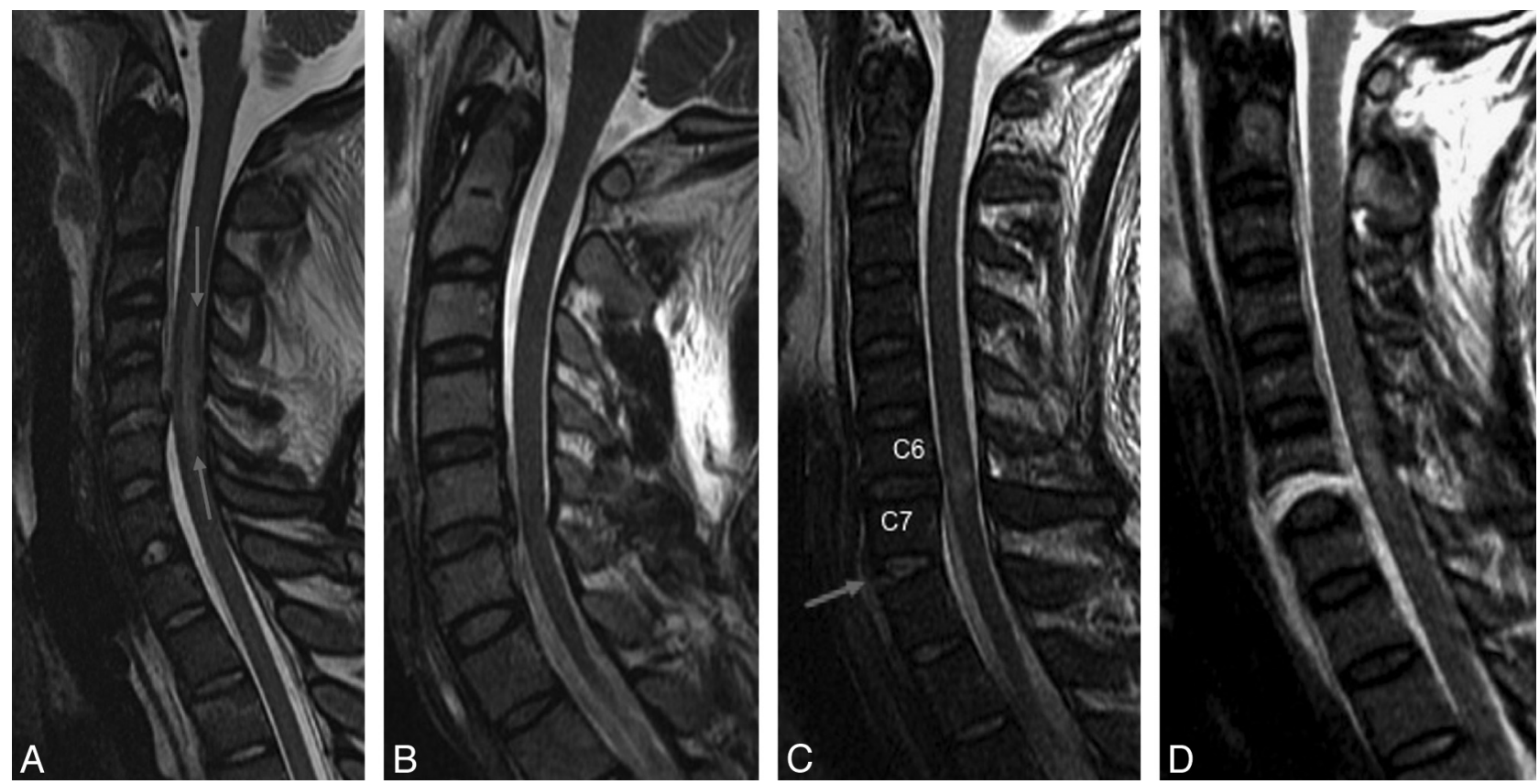

FIGURE. A, Traumatic herniation disk producing spinal cord compression at the same level; it is seen as hyperintensity in the T2-weighted image. Lesion length is measured in millimeters from the most cephalad level to the lowest (arrows). B, Sagittal projection on T2-weigthed images. Disruption of posterior longitudinal ligament is visualized at level C5-C6. C, Sagittal projection on T2-weighted image. At level C7-T1, the injured anterior longitudinal ligament (arrow) is seen. Signal changes are shown at level C6-C7. D, Damage in anterior longitudinal ligament, posterior longitudinal ligament, ligamentum flavum, and disk are represented by a disruption in low signal intensity corresponding to this structure.

lesions, which MR imaging can detect, including more severe SCI. Soft-tissue injury is a more static finding than is cord edema and can convey potentially relevant information when MR imaging is delayed (Fig 1).

\section{MATERIALS AND METHODS}

Patients with known SCI after non-penetrating cervical spine trauma over a 21-year period were recruited in the clinical, retrospective data base. Only patients in whom cervical MR imaging was performed within 96 hours after trauma were selected for further analysis.

Patients who were neurologically intact, or whose MR imaging was performed after the fourth day of the injury, were excluded. Patients with severe traumatic brain injury or upper cervical spine injuries (C1-C2 fractures) were also excluded from the analysis. Thoracic injuries were not included either.

We recorded clinical variables, including age, sex, mechanism of injury, and neurologic status. Epidemiologic data also examined age, sex, and mechanism of injury from the medical records. Neurologic status was assessed clinically during the first examination by using the American Spinal Injury Association Impairment Scale (ASIA) (Table 1).

Plain radiographs, CT scans, and MR images were consensually assessed by a neurosurgeon trained in cervical trauma images (I.P.) and an experimental neuroradiologist (A.R.). Both observers were blinded to patient clinical and neurologic data.

To diagnose the level and extension of injury and determine the number of fractures, plain radiographs, MR imaging, and CT were used. The presence of fracture and bone integrity was assessed in the CT scan.

Soft-tissue injury was assessed with the use of MR imaging and

\section{Table 1: American Spinal Injury Association Impairment Scale}

Grade A Complete: No sensory or motor function is preserved in the sacral segments S4-S5

Grade B Incomplete: Sensory but not motor function is preserved below the neurological level and includes the sacral segments S4-S5

Grade C Incomplete: Motor function is preserved below the neurological level, and more than half of key muscles below the neurological level have a muscle grade $<3$

Grade D Incomplete: Motor function is preserved below the neurological level, and at least half of key muscles below the neurological level have a muscle grade $\geq 3$

Grade E Normal: Sensory and motor function is normal

analyzed with anterior longitudinal ligament (ALL), posterior longitudinal ligament (PLL), and ligamentum flavum (LF), and the intervertebral disk was examined. In all patients, MR imaging was performed within the first 4 days after trauma. MR imaging was performed with the use of a $1.5 \mathrm{~T}$ magnet and 3-mm section thickness for axial and sagittal planes. We analyzed ligamentous injury by assessing disruption in $\mathrm{T} 1$ - and $\mathrm{T} 2$-weighted images (in a sagittal plane), including axial T2- and $\mathrm{T} 2{ }^{*}$-weighted images. In all patients, lesion length was determined by intramedullary cord signal intensity change in T2-weighted images and was defined as the distance in millimeters between the most caudal and cephalad signal intensity change in the cord. Soft-tissue disruption was defined as a clear MR imaging signal with complete discontinuity of the structure in all appropriate imaging sections.

The segment affected was defined as fracture level with only 1 fracture: if there was more than 1 , it was defined as the fracture 
level that produced the most cord compression. In cases with SCI but without radiologic abnormalities (SCIWORA), we considered the segment to be affected when the soft tissues were most injured.

We analyzed the data with univariate and multivariate methods to compare the damage of soft-tissue structures (LF, ALL, PLL, and disk), including the extent of SCI. ALL, PLL, LF, and disk injuries were independently analyzed as dichotomous variables (damaged or intact); the extent of SCI (distance between the most caudal and cephalad of the cord signal intensity change [hyperintensity] in a sagittal plane, T2-weighted image measured in millimeters) was considered to be a continuous variable. Levels of injury, age, and sex were analyzed as potential covariates. Sex (male/female) and level of injury (upper lesion, C3-C4-C5 and lower lesion, C6-C7-C8) were considered dichotomous variables, whereas age was seen as a continuous variable. The Mann-Whitney $U$ test compared lesion length of each predefined qualitative variable (ALL, PLL, LF, and disk damage, sex, and level of injury). Spearman $\rho$ was calculated to demonstrate the correlation between lesion length and age. Variables with a value of $P<.1$ were included in the multivariate analysis. Linear regression was used for the multivariate analysis as well.

The Kruskall-Wallis test could also probe a possible association between how many ligaments were affected (none, 1, 2, or 3 ligaments), including lesion length.

The association between lesion length and neurologic status was also analyzed. Lesion length was considered a continuous variable, as stated previously. Neurologic status at admission was separated as complete SCI (ASIA A) and incomplete SCI (ASIA B, $\mathrm{C}$, and D). We used a $t$ test to compare the lesion length for the 2 groups. The threshold for statistical significance was $P<.05$, and all tests were calculated by use of SPSS v20 (IBM, Armonk, New York).

\section{RESULTS}

We retrospectively reviewed 331 patients with known or suspected SCI or with radiculopathy after a non-penetrating cervical spine trauma who presented to the emergency department or the intensive care unit at our institution between 1990-2011. MR imaging was performed on 186 patients within 96 hours after trauma who were then selected for further analysis. We excluded 40 patients with severe traumatic brain injury from the analysis. In addition, 38 patients with upper cervical spine trauma (C1-C2 fractures) were also excluded. Finally, data analysis included 108 patients ( 87 men and 21 women, ages $17-86$ years; overall mean age, 40.5 years) (Table 2). Seventy-four patients had 1 fracture, 13 patients had 2, and 3 patients had 3 noncontiguous fractures, respectively. In 18 patients, there was no evidence of any fracture after radiologic examination: all patients were diagnosed with SCI without radiologic abnormalities (SCIWORA). The most common mechanism of injury was motor vehicle crash (Table 2). The C5-C6 spinal level was most commonly involved (Table 2). As stated, MR imaging was performed within 96 hours after the trauma occurred in all patients, whereas in $81.4 \%$ of these patients, it was performed within the first 72 hours. According to ASIA, there were 39 patients with ASIA A, 10 patients with ASIA B, 22 patients with ASIA C, and 37 patients with ASIA D (Table 2).
Table 2: Characteristics of patients with acute traumatic cervical SCI

\begin{tabular}{lc}
\hline \multicolumn{1}{c}{ Characteristic } & No. of Patients (\%) \\
\hline Mean age, y & $40.5(17-86)$ \\
Male & $87(80.6)$ \\
Female & $21(19.4)$ \\
Severity of SCI & \\
ASIA A & $39(36.1)$ \\
ASIA B & $10(9.3)$ \\
ASIA C & $22(20.3)$ \\
ASIA D & $37(34.3)$ \\
Spinal level of SCI & \\
C3-C4 & $16(14.8)$ \\
C4-C5 & $22(20.3)$ \\
C5-C6 & $31(28.7)$ \\
C6-C7 & $24(22.2)$ \\
C7-T1 & $15(13.8)$ \\
Cause of SCI & \\
Motor vehicle crash & $61(56.4)$ \\
Car crash & $35(32.4)$ \\
Pedestrians & $8(7.4)$ \\
Motorcyclists & $18(16.6)$ \\
Falls & $33(30.6)$ \\
Sport injury & $9(8.3)$ \\
Aggression/assault & $5(4.6)$ \\
\hline
\end{tabular}

Table 3: Univariate analysis: Qualitative variables (soft tissues)

\begin{tabular}{|c|c|c|c|c|c|}
\hline \multirow[b]{2}{*}{ Structure } & \multicolumn{2}{|c|}{ No. of Cases (\%) } & \multicolumn{2}{|c|}{$\begin{array}{l}\text { Mean Length of } \\
\text { Lesion, } \mathrm{mm}\end{array}$} & \multirow{2}{*}{$\begin{array}{c}P \\
\text { Value }\end{array}$} \\
\hline & Intact & Disrupted & Intact & Disrupted & \\
\hline ALL & $51(47.2)$ & $57(52.8)$ & 43.22 & 64.60 & $<.001$ \\
\hline PLL & 45 (41.7) & $63(58.3)$ & 47.64 & 59.40 & .05 \\
\hline LF & $56(51.9)$ & $52(48.1)$ & 35.96 & 74.46 & $<.001$ \\
\hline Disk & $46(42.6)$ & $62(57.4)$ & 47.48 & 59.71 & .044 \\
\hline
\end{tabular}

Table 4: Multivariate analysis: Linear regression

\begin{tabular}{ccc}
\hline Dependent Variable & Independent Variable & $\boldsymbol{P}$ Value \\
\hline Lesion length $(\mathrm{mm})$ & ALL & .395 \\
& PLL & .525 \\
& LF & $<.001$ \\
& Disk & .464 \\
& Age & .215 \\
\hline
\end{tabular}

Note:- $R^{2}$ value of prediction model number $1=0.365$.

Age was correlated with lesion length $(P=.036)$ in the univariate analysis and was included in the multivariate analysis as a covariate. The level of lesion $(P=.449)$ and sex $(P=.398)$ were not associated with larger lesions in the univariate analysis. Eighteen patients did not show hyperintensity changes in T2-weighted images. ALL was disrupted in $52.8 \%$ of cases, PLL in $58.3 \%$, LF in $48.1 \%$, and disk in $57.4 \%$. Average cord lesion length (in $\mathrm{mm}$ ) for disrupted and non-disrupted soft tissue was 64.60 and 43.22 for ALL, 59.40 and 47.64 for PLL, 74.46 and 35.96 for LF, 59.71 and 47.48 for disk, respectively. All structures showed a greater mean lesion length when there was soft-tissue disruption, compared with when there was not disruption. With the use of univariate analysis, LLA, PLL, LF, and disk disruption were related to a significantly larger lesion length (Table 3). However, in multivariate analysis, LF was the only structure showing a significant association between the ligament injured and greater lesion length (Table 4).

There were 22 patients without disrupted ligaments, 34 with 1 
disrupted ligament, 17 with 2 disrupted ligaments, and 35 with 3 disrupted ligaments. Mean lesion lengths in millimeters were $29.75,50.15,56.38$, and 73.37 for none, 1, 2, and 3 disrupted ligaments, respectively. The number of disrupted ligaments correlated with lesion length (Pearson correlation coefficient, 0.49; $P<.01$ ).

Patients with complete SCI at admission had a larger intramedullary lesion $(P<.001)$ than did patients with incomplete SCI.

\section{DISCUSSION}

Previous studies show that lesion lengths measured in sagittal T2-weighted images are related to patient prognosis. ${ }^{9,12-14}$ Edema is seen in T2 MR imaging sequences as hyperintensity of the signal within the cord, whereas a low intensity area on T2-weighted images in the acute stage is thought to indicate intramedullary hemorrhage, attributed to deoxyhemoglobin. ${ }^{15,16}$ In addition, because hemorrhage is almost always concurrent with edema, it is common to measure signal hyperintensity in T2-weighted images to determine the length of the lesion. ${ }^{17}$ It is not clear whether these signal changes are a result of primary or secondary spinal cord damage. ${ }^{18}$

MR imaging may be a useful tool in the management of cervical trauma, especially in cases of questionable structural instability, because it is extremely useful in detecting soft-tissue injury. ${ }^{19,20}$ Patients without demonstrated abnormalities in plain radiographs or CT may show signs of instability with MR imaging, as determined by soft-tissue involvement. Some authors have demonstrated that different ligamentous injuries are associated with spinal instability; therefore, this could serve as a guide for surgical treatment of traumatic instability of the spinal cord. Greater posterior vertebral body translation and angulation are related to disruption of the posterior ligamentous complex ${ }^{21,22}$ and are indirect signs of spinal instability.

For assessing disruption of ligaments, T2-weighted images in the sagittal plane should be included in all MR imaging protocols. ${ }^{3}$ Other sequences have been described in the literature to rule out soft-tissue injuries, such as fat-suppressed T2 images (STIR). ${ }^{19,20}$ In acute cervical trauma, MR imaging has moderate to high sensitivity for injury of specific ligamentous structures but low specificity for intraoperative findings of the same injuries. ${ }^{23,24}$ The posterior ligamentous complex consists of supraspinous and interspinous ligaments: the LF, the facet capsules, and the cervical fascia. ${ }^{25-28}$ In several studies, MR imaging was also sensitive for the evaluation of injury of the posterior ligamentous complex $^{19,24}$; this suggests that injury to ALL may be underestimated with the use of MR imaging, if the findings are considered in isolation. The explanation is that ALL and PLL adhere to the disk, whereas only the ALL adheres to the vertebrae, such that the PLL is easier to identify. ${ }^{3}$

We have discussed the importance of MR imaging in the detection of ligamentous injury after cervical trauma: it also determines the structural stability of the cervical spine. Spinal instability is related to neurologic injury and therefore to cord injury because the involvement of soft tissue may play a role in the extent of SCI. This report shows that damage to soft tissue such as LF, ALL, PLL, and disk is related to longer-length cord hyperintensity as measured in T2-weighted images; when this was adjusted for age, level of injury, sex, and ligamentous or disk injury in a multivariate analysis, only LF injury was found to be significantly associated with lesion length.

We hypothesize that LF injury is more strongly correlated with extension of cord damage, in that posterior elements are associated with increased instability and therefore with greater SCI. Another possible explanation is that the LF is more elastic and that more pressure is needed to break it. The force required for this kind of injury would be responsible for SCI; it is probably the result of a combination of the 2 mechanisms because they are not mutually exclusive. We excluded patients with upper cervical fractures because these lesions are the result of different injury mechanisms. ${ }^{18}$

Several prognostic factors have been described as predictive of traumatic spinal cord damage. ${ }^{10,13,17,29-32}$ Some studies have focused on particular clinical features, such as SCIWORA, ${ }^{29,33}$ Central Cord syndrome, ${ }^{29}$ or Brown-Sequard syndrome. ${ }^{34}$ Evidence in the literature shows that more severe abnormalities seen through MR imaging have been associated with more severe neurologic status. Excessive edema, changes in T2-weighted images, greater degree of cord compression, and hemorrhage have been related to worse neurologic outcomes. ${ }^{9,35-38}$ Gain in motor strength at follow-up has been seen to correlate with a decrease in T2-weighted hyperintensity in serial MR imaging studies. ${ }^{39}$ Some authors have described a close correlation between level of injury and severity of SCI. ${ }^{30}$ Miranda et $\mathrm{al}^{34}$ found an association between MR imaging signal changes and level of injury. Therefore, because the level of injury is related anatomically with the level of fracture, we also introduced this factor in the multivariate analysis. To our knowledge, there is 1 paper in the literature that relates the severity of ligamentous injury with degree of SCI. ${ }^{6}$ In this study, we found that the severity of PLL, versus ALL injury, was correlated with more severe SCI, especially in patients with extension fractures.

Our study found that patients without signal changes in the spinal cord had few neurologic deficits. Patients with complete SCI showed larger lesions than did those with incomplete SCI. This in turn highlights the correlation between radiologic and clinical findings.

\section{Limitations}

The first limitation of our study may be related to the timing of the MR imaging. Clinical instability associated with cervical trauma sometimes impedes the reliability of MR imaging in the first hours. A review of the literature by Bozzo et $\mathrm{al}^{3}$ concluded that MR imaging should be done in the acute period after SCI for better prognostication. It has also been recommended that the first MR imaging should be performed 24-72 hours after trauma. ${ }^{10}$ However, there is a lack of evidence supporting precise guidelines. ${ }^{3,23}$ In fact, transferring critical or unstable patients from the intensive care unit to an $\mathrm{x}$-ray room presents an unacceptable risk during the first days, especially in patients with trauma. Length of edema in T2-weighted images depends on the time when the MR imaging was performed after trauma. ${ }^{11}$ The extension of the lesion may be overestimated in patients in whom MR imaging was performed at a later point in time. To focus on 
acute traumatic injury, and to minimize potential bias introduced with chronic injury, we only included patients who had undergone MR imaging during the first 96 hours after injury. In 82.4\% of these patients, MR imaging was performed within 72 hours after trauma.

The second limitation of our study could be correct identification and diagnosis of ligament disruption. T2-weighted images have shown moderate to high sensitivity with low specificity when compared with intraoperative findings in ligament disruption. ${ }^{23,24}$ Other sequences have been described in the literature that rule out soft-tissue injuries, such as STIR. One study showed a high correlation between STIR sequences and intraoperative findings in detecting soft-tissue injury. ${ }^{27}$ Unfortunately, the STIR sequence was not included in all cervical trauma protocols during the initial years of this study; in this sense, not all patients were observed for STIR sequence, even though most were. We used STIR for assessment of soft tissue when it was available. Any assessment of the extent of damage in spinal cord and ligament injuries is subjective, which poses another potential limitation and source of bias.

Third, this study comprised a select group of cases that were managed in the intensive care or neurosurgery units. Minor cervical trauma with a lesser degree of lesion does not require MR imaging by protocol in most cases. Furthermore, this study does not include all patients with cervical spine trauma, but it does involve most patients with more severe cervical trauma.

Fourth, steroids were not included in our treatment protocol after Bracken et al ${ }^{40}$ could not corroborate statistically significant benefits from steroids. Most patients did not receive steroids, and it was therefore not possible to access this information in relation to the time of imaging; thus, this factor could not be adequately analyzed because it could have affected length of abnormal cord signals.

Finally, lesion length represents the degree of SCI but is not a measure of neurologic outcome at follow-up. In this light, interpretation of these results should be taken with caution. Nevertheless, initial neurologic examination tends to have a certain degree of subjectivity: in patients with trauma, sedation, distractive pain, or clinical condition confounds reliable interpretation of the neurologic state at admission. This is often the case when it is necessary to differentiate between ASIA A (complete SCI) and ASIA B (incomplete, but no motor function preserved) in sedated patients. In the acute phase, lesion length is a measure that has been well correlated with neurologic recovery. ${ }^{9,11}$ Reliability issues, as the result of feasibility of clinical examination for initial assessment of cervical spine trauma, were better determined in our study when MRI was performed within 96 hours after trauma. We prefer to use a continuous quantitative variable to indirectly appraise the degree of SCI. Our results correlate with those of previous works, ${ }^{8,9,36}$ and larger lesions statistically correlate with more severe neurologic deficits with complete SCI in the acute phase $(P<.001)$. However, other investigators have found that lesion length depends on the time of MR imaging after trauma. ${ }^{11}$ The value of the extent of the lesion may be overestimated in patients for whom MR imaging was performed later. Therefore, we must determine which soft-tissue injury is associated with a greater degree of SCI, given that this is a static finding; however, intramedullary signal changes are dynamic, and their prognostic value decreases over time. Injury of the LF is a feature perdurable over time. It can also be detected when MR imaging is performed at a more delayed stage if the patient is clinically unstable during the acute phase and the MR imaging is not able to be obtained at an earlier stage. It could also be helpful in predicting neurologic outcome in these cases.

\section{CONCLUSIONS}

We have presented the first study that correlates ALL, PLL, LF, and disk lesions according to the degree of spinal cord damage. Patients who had disruption of the LF showed greater length of lesion as measured by MR imaging. Additionally, the statistical association between number of ligaments injured and greater signal changes in T2-weighted images of the spinal cord has been demonstrated.

We still have several concerns (timing of imaging, poor specificity in measurement method, short follow-up period, lack of objectivity); however, findings suggest that the extent of the T2 signal (after cervical spine trauma) correlates to a specific pattern of ligamentous injury. This might help determine neurologic outcome when MR imaging is performed after the acute phase, in that other features have less predictive potential.

Carefully designed studies, including neurologic outcome at follow-up, are needed to confirm some of our preliminary observations.

Disclosures: Ana Ramos—UNRELATED: Grants/Grants Pending: FIS* (*money paid to institution).

\section{REFERENCES}

1. Hasler RM, Exadaktylos AK, Bouamra O, et al. Epidemiology and predictors of spinal injury in adult major trauma patients: European cohort study. Eur Spine J 2011;20:2174-80

2. Milby AH, Halpern $\mathrm{CH}$, Guo W, et al. Prevalence of cervical spinal injury in trauma. Neurosurg Focus 2008;25:E10

3. Bozzo A, Marcoux J, Radhakrishna M, et al. The role of magnetic resonance imaging in the management of acute spinal cord injury. J Neurotrauma 2011;28:1401-11

4. Boldin C, Raith J, Fankhauser F, et al. Predicting neurologic recovery in cervical spinal cord injury with postoperative MR imaging. Spine 2006;31:554-59

5. Pizones J, Izquierdo E, Sanchez-Mariscal F, et al. Sequential damage assessment of the different components of the posterior ligamentous complex after magnetic resonance imaging interpretation: prospective study 74 traumatic fractures. Spine 2012;37:E662-67

6. Song KJ, Kim GH, Lee KB. The efficacy of the modified classification system of soft tissue injury in extension injury of the lower cervical spine. Spine 2008;33:E488-93

7. Vaccaro AR, Madigan L, Schweitzer ME, et al. Magnetic resonance imaging analysis of soft tissue disruption after flexion-distraction injuries of the subaxial cervical spine. Spine 2001;26:1866-72

8. Flanders AE, Spettell CM, Tartaglino LM, et al. Forecasting motor recovery after cervical spinal cord injury: value of MR imaging. $R a$ diology 1996;201:649-55

9. Miyanji F, Furlan JC, Aarabi B, et al. Acute cervical traumatic spinal cord injury: MR imaging findings correlated with neurologic outcome: prospective study with 100 consecutive patients. Radiology 2007;243:820-27

10. Bondurant FJ, Cotler HB, Kulkarni MV, et al. Acute spinal cord injury: a study using physical examination and magnetic resonance imaging. Spine 1990;15:161-68

11. Leypold BG, Flanders AE, Burns AS. The early evolution of spinal 
cord lesions on MR imaging following traumatic spinal cord injury. AJNR Am J Neuroradiol 2008;29:1012-16

12. Andreoli C, Colaiacomo MC, Rojas Beccaglia M, et al. MRI in the acute phase of spinal cord traumatic lesions: relationship between MRI findings and neurological outcome. Radiol Med 2005;110: 636- 45

13. Ramon S, Dominguez R, Ramirez L, et al. Clinical and magnetic resonance imaging correlation in acute spinal cord injury. Spinal Cord 1997;35:664-73

14. Shimada K, Tokioka T. Sequential MR studies of cervical cord injury: correlation with neurological damage and clinical outcome. Spinal Cord 1999;37:410-15

15. Flanders AE, Schaefer DM, Doan HT, et al. Acute cervical spine trauma: correlation of MR imaging findings with degree of neurologic deficit. Radiology 1990;177:25-33

16. Kulkarni MV, Bondurant FJ, Rose SL, et al. 1.5 Tesla magnetic resonance imaging of acute spinal trauma. Radiographics 1988;8: $1059-82$

17. Flanders AE, Spettell CM, Friedman DP, et al. The relationship between the functional abilities of patients with cervical spinal cord injury and the severity of damage revealed by MR imaging. AJNR Am J Neuroradiol 1999;20:926-34

18. Alday R, Lobato, RD, Gomez PA. Cervical spine fractures. In: Neurosurgery. New York: Churchill Livingstone; 1996

19. Pizones J, Sanchez-Mariscal F, Zuniga L, et al. Prospective analysis of magnetic resonance imaging accuracy in diagnosing traumatic injuries of the posterior ligamentous complex of the thoracolumbar spine. Spine 2013;38:745-51

20. Pizones J, Zuniga L, Sanchez-Mariscal F, et al. MRI study of posttraumatic incompetence of posterior ligamentous complex: importance of the supraspinous ligament. prospective study of 74 traumatic fractures. Eur Spine J 2012;21:2222-31

21. Radcliff K, Su BW, Kepler CK, et al. Correlation of posterior ligamentous complex injury and neurological injury to loss of vertebral body height, kyphosis, and canal compromise. Spine 2012;37: $1142-50$

22. Samartzis D, Wein SM, Shen FH, et al. A revisitation of distractiveextension injuries of the subaxial cervical spine: a cadaveric and radiographic soft tissue analysis. Spine 2010;35:395-402

23. Goradia D, Linnau KF, Cohen WA, et al. Correlation of MR imaging findings with intraoperative findings after cervical spine trauma. AJNR Am J Neuroradiol 2007;28:209-15

24. Rihn JA, Fisher C, Harrop J, et al. Assessment of the posterior ligamentous complex following acute cervical spine trauma. $J$ Bone Joint Surg Am 2010;92:583-89

25. Carrino JA, Manton GL, Morrison WB, et al. Posterior longitudinal ligament status in cervical spine bilateral facet dislocations. Skeletal Radiol 2006;35:510-14

26. Haba H, Taneichi H, Kotani Y, et al. Diagnostic accuracy of magnetic resonance imaging for detecting posterior ligamentous complex injury associated with thoracic and lumbar fractures. J Neurosurg 2003;99:20-26

27. Lee HM, Kim HS, Kim DJ, et al. Reliability of magnetic resonance imaging in detecting posterior ligament complex injury in thoracolumbar spinal fractures. Spine 2000;25:2079-84

28. Vaccaro AR, Hulbert RJ, Patel AA, et al. The subaxial cervical spine injury classification system: a novel approach to recognize the importance of morphology, neurology, and integrity of the disco-ligamentous complex. Spine 2007;32:2365-74

29. Collignon F, Martin D, Lenelle J, et al. Acute traumatic central cord syndrome: magnetic resonance imaging and clinical observations. J Neurosurg 2002;96:29-33

30. Liao CC, Lui TN, Chen LR, et al. Spinal cord injury without radiological abnormality in preschool-aged children: correlation of magnetic resonance imaging findings with neurological outcomes. J Neurosurg 2005;103:17-23

31. Silberstein M, Hennessy O. Implications of focal spinal cord lesions following trauma: evaluation with magnetic resonance imaging. Paraplegia 1993;31:160-67

32. Yamashita $Y$, Takahashi M, Matsuno $Y$, et al. Acute spinal cord injury: magnetic resonance imaging correlated with myelopathy. Br J Radiol 1991;64:201-09

33. Dare AO, Dias MS, Li V. Magnetic resonance imaging correlation in pediatric spinal cord injury without radiographic abnormality. J Neurosurg 2002;97:33-39

34. Miranda P, Gomez P, Alday R, et al. Brown-Sequard syndrome after blunt cervical spine trauma: clinical and radiological correlations. Eur Spine J 2007;16:1165-70

35. Dai L, Jia L. Central cord injury complicating acute cervical disc herniation in trauma. Spine 2000;25:331-35

36. Marciello MA, Flanders AE, Herbison GJ, et al. Magnetic resonance imaging related to neurologic outcome in cervical spinal cord injury. Arch Phys Med Rehabil 1993;74:940-46

37. Selden NR, Quint DJ, Patel N, et al. Emergency magnetic resonance imaging of cervical spinal cord injuries: clinical correlation and prognosis. Neurosurgery 1999;44:785-92

38. Shepard MJ, Bracken MB. Magnetic resonance imaging and neurological recovery in acute spinal cord injury: observations from the National Acute Spinal Cord Injury Study 3. Spinal Cord 1999; 37:833-37

39. Miranda P, Gomez P, Alday R. Acute traumatic central cord syndrome: analysis of clinical and radiological correlations. J Neurosurg Sci 2008;52:107-12

40. Bracken MB, Shepard MJ, Collins WF, et al. A randomized, controlled trial of methylprednisolone or naloxone in the treatment of acute spinal-cord injury: results of the Second National Acute Spinal Cord Injury Study. N Engl J Med 1990;322:1405-11 